\title{
Cultura empresarial y rendimiento de la PYME en el estado de Aguascalientes (México)
}

\author{
Domingo García Pérez de Lema* \\ Martha González Adame** \\ Salvador Vivanco Florido** \\ * Universidad Política de Cartagena, España \\ domingo.garcia@upct.es \\ ** Universidad Autónoma de Aguascalientes (México) \\ mgonzale@correo.uaa.mx \\ svivanco@correo.uaa.mx
}




\section{RESUMEN}

La cultura empresarial es un factor determinante para alcanzar el éxito competitivo de las organizaciones. Este trabajo analiza el tipo de cultura implementado en las empresas y su relación con el rendimiento. Para llevar a cabo este análisis se ha realizado un estudio empírico con una muestra de 400 micro, pequeñas y medianas empresas (mPyme) del estado de Aguascalientes (México). Los resultados muestran que la cultura predominante en las empresas es la de tipo clan. Y encontramos evidencia de que existe una relación positiva entre la cultura clan y el rendimiento de la empresa, mientras que surge una relación negativa con la cultura jerárquica.

Palabras clave: cultura empresarial, cultura organizacional, rendimiento, Pyme.

Clasificación JEL: M19, M29

\section{ABSTRACT}

The enterprise culture is a determining factor to achieve the competitive success of the organizations. This work analyzes the type of culture implemented in the companies and their relation with the performance. In order to carry out this analysis an empirical study has been made with a sample of 400 SME of Aguascalientes (Mexico). The results show that the predominant culture in the companies is the one of type clan. And we found evidence that a positive relation between the culture clan and the yield of the company exists, whereas arises a negative relation with the hierarchic culture.

Keywords: Organizational Culture, Corporate Culture, Performance, Sme

JEL Clasification: M19, M29

Revista de Economía, vol. XXVII, núm. 75, segundo semestre de 2010. 


\section{INTRODUCCIÓN}

El cambio se está institucionalizando como una práctica requerida en todas las organizaciones, con independencia de su tamaño, sector de actividad, sistema técnico e incluso nivel de internacionalización. Son varios los factores que les están obligando a estar continuamente llevando a cabo variaciones en sus productos y servicios, en sus procesos y en sus sistemas de gestión. Entre ellos, el aumento de la competencia, el corto ciclo de vida de los productos y la inestabilidad económica. Esta situación les obliga a introducir de forma estable innovaciones.

La cultura empresarial es un factor clave que puede ayudar a las empresas a mejorar la competitividad de la empresa. Si los directivos cambian los valores, normas y costumbres de la empresa, podrían modificar el comportamiento y aptitud de sus empleados, mostrando una mejora del rendimiento de la empresa (Rosenthal y Masarech, 2003).

Actualmente las organizaciones no se plantean tanto si deben de cambiar o no, sino "cómo" cambiar para conseguir mejorar su eficacia y eficiencia, en definitiva, sus resultados operativos y financieros. La implantación de sistemas de calidad total, el análisis de procesos, la reingeniería, la reducción de plantillas (downsizing), el aumento de equipos multifuncionales cuentan entre las prácticas que las organizaciones están poniendo en marcha para hacer frente a la incertidumbre actual. Son numerosos los casos donde no se han conseguido implantarlas de forma exitosa.

En opinión de Cameron y Quin (1999) el motivo de este fracaso se debe a que la mayoría de las empresas han procedido únicamente a implantar la técnica concreta sin modificar la cultura de la organización. Es difícil que se observen mejoras en los resultados tras la introducción del cambio si se siguen manteniendo el mismo conjunto de valores, normas y costumbres, ya que pronto el personal volverá a actuar igual que lo hacía antes de la nueva situación.

La cultura empresarial es un factor clave que puede ayudar a las compañías a mejorar su rendimiento. La cultura es lo que distingue realmente 
a las organizaciones de alto rendimiento (Jeuchter et al. 1998) y se convierte en un tópico de investigación de primer orden. Investigaciones anteriores en otros contextos económicos (Siehl y Martin, 1998; Denison y Spreitzer, 1991; Deal y Kennedy, 1982; Maani, Puga y Stuti, 1994; Petty, Bedeles y Lowery, 1995) han revelado que existe una posible relación entre la cultura organizacional y el rendimiento.

El objetivo de este trabajo es, por una parte, tipificar el tipo de cultura empresarial que existe en el seno de las Pyme en México, siguiendo la tipología de Cameron y Quinn (1999); y por otra parte, analizar la relación existente entre los diferentes tipos de cultura empresarial y el rendimiento de la Pyme. Para ello se realiza un estudio empírico a través de una muestra de 400 Pyme del Estado de Aguascalientes (México). ${ }^{1}$

El trabajo se ha estructurado de la siguiente forma: en primer lugar, se determina el marco teórico, se realiza una revisión de la literatura sobre el marco de la cultura empresarial y su relación con el rendimiento; en segundo lugar, se expone la metodología, las características de la muestra y la justificación de las variables utilizadas; en tercer lugar, se lleva a cabo el análisis de los resultados, y finalmente, se exponen las principales conclusiones alcanzadas.

\section{CULTURA EMPRESARIAL Y RENDIMIENTO}

Con el término "cultura empresarial”, o el más amplio, "cultura organizativa", se hace referencia al "conjunto de creencias, expectativas y principios fundamentales o básicos, compartidos por los miembros de una organización. Estas creencias y expectativas producen reglas de conducta (normas) que configuran poderosamente la conducta de los individuos y grupos de la organización, y de esta forma la diferencia de otras organizaciones" (Leal, 1991).

\footnotetext{
${ }^{1}$ Este trabajo se inserta en un proyecto de investigación más amplio desarrollado en la Universidad Autónoma de Aguascalientes dirigido por García y Martínez (2009) con el título Innovación y cultura empresarial de las MIPYME (Estado de Aguascalientes).
} 
En su aspecto más profundo la cultura está formada por valores, esto es, "las preferencias integradas y tácitas acerca de los objetivos que la organización debe alcanzar y de cómo debería hacerlo" (De Long y Fahey, 2000). Los valores son los que van a determinar las normas y las costumbres. Estas últimas son "expectaciones creadas socialmente sobre las actitudes y comportamientos que se consideran aceptables en la organización" (Tushman y O’ Reilly III, 2002). La cultura, de esta forma, actúa como sistema social de control ya que, si la cultura es sólida, aquellos que comparten las normas llamarán la atención sobre los que las están violando.

Como se observa, la cultura refleja los valores, normas y costumbres realmente existentes y no aquellas que a la dirección le gustaría que existieran. Muchos empresarios no son conscientes de que "la cultura empresarial” es un factor más, que sabiéndolo gestionar puede ayudarles a alcanzar los objetivos que pretenden.

La importancia que tiene para cualquier director actuar sobre la cultura de su organización se deriva del hecho de que cambiando los valores, las normas y las costumbres realmente existentes se consiguen modificar los comportamientos y las actitudes de los individuos dentro de su organización, y de esta forma se pueden mejorar los resultados operativos, lo cual tendrá un efecto positivo en los resultados financieros.

La relación anteriormente expresada debe ser vista tanto desde un punto de vista estático como dinámico. Esto es, a través de la gestión de la cultura empresarial las organizaciones pueden mejorar su situación actual consiguiendo aquellos valores, normas y costumbres requeridos para el éxito de nuestros productos y servicios actuales.

Debido a la incertidumbre ambiental existente, las organizaciones también pueden y deben utilizar la gestión de la cultura para desarrollar una actitud proactiva. De hecho, muchas organizaciones necesitan estar mejorando lo que hacen y, a la vez, estar probando e introduciendo productos y servicios nuevos. Esto es, las organizaciones requieren estar continuamente innovando. 
Una de las hipótesis de investigación más estudiadas se basa en la relación que existe entre la presencia de una cultura implantada y su efecto sobre la rentabilidad de la empresa (Rosenthal y Masarech, 2003). Este efecto positivo de la cultura sobre los resultados de la empresa, se basa en la idea de que tener trabajadores motivados con base en la existencia de una fuerte cultura empresarial conlleva a la consecución de unos objetivos comunes a todos (Kotter y Heskett, 1992).

Así, determinados estudios empíricos han demostrado que las compañías con una fuerte cultura consiguen un mayor beneficio que aquellas con una débil cultura (Kotter y Heskett, 1992; Gordon y Di Tomaso, 1992; Burt y Sparks, 1994). En este sentido, Kotter y Heskett (1992) comprobaron que aquellas empresas que habían desarrollado una cultura obtenían mejores resultados que las que no lo habían hecho.

Por otra parte, Sorensen (2002) contrastó que aquellas empresas con una cultura empresarial implantada tenían una mejor capacidad de adaptación a las condiciones de un entorno cambiante. Así, una fuerte cultura organizativa facilita la estabilidad del beneficio siempre que el entorno sea relativamente estable, aunque conforme se incrementa la volatilidad las ventajas de la cultura decrecen.

Adicionalmente, hay estudios realizados por Denison y Mishra (1995) que han contribuido significativamente al campo de la cultura y el rendimiento, donde la cultura está siendo considerada como una variable de investigación. Denison y Mishra (1995) descubrieron que la cultura se correlacionó significativamente con el rendimiento financiero a corto plazo; Schneider (1990) también encontró que las organizaciones centradas en la cultura son más exitosas, porque centrarse en ella proporciona mejores rendimientos financieros, que incluyen alto rendimiento de la inversión, de activos y de fondos propios. Estos hallazgos han avanzado en la comprensión de los determinantes y efectos del rendimiento con la cultura corporativa.

Esta conducta está en consonancia con el patrón de comportamiento señalado por March (1991), al sugerir que aquellas empresas con una fuerte cultura son extremadamente buenas en aprovechar las ventajas 
competitivas que ya tienen, pero encuentran dificultades para explorar y descubrir nuevas ventajas que se adapten mejor al entorno cambiante. Gordon y Di Tomaso (1992) defienden que la mejor estrategia de empresa consiste en desarrollar una cultura sostenible que se base en el aprendizaje exploratorio y la innovación.

Con base en estos argumentos la hipótesis que se plantea es la siguiente:

$\mathrm{H}_{1}$ : La cultura empresarial implantada en la empresa influye significativamente en su rendimiento.

\section{METODOLOGÍA DE LA INVESTIGACIÓN}

\subsection{La muestra}

Los datos se obtuvieron del proyecto de investigación "Innovación y cultura empresarial de la MiPyme del estado de Aguascalientes" dentro del convenio de colaboración entre la Universidad Autónoma de Aguascalientes (México), la Universidad Politécnica de Cartagena, la Universidad de Murcia y la Universidad de Cantabria (España).

Para determinar la muestra, se trabajó con los datos proporcionados por el directorio empresarial del Sistema de Información Empresarial de México (SIEM) 2008, de la Secretaría de Economía (SE), el cual tenía 8,661 empresas registradas al 31 de agosto de 2008, de todos los sectores y de todos los tamaños. Para efectos de este estudio, se tomaron únicamente aquellas empresas que tenían entre 5 y 250 trabajadores (Micros, Pequeñas y Medianas empresas) del estado de Aguascalientes, con lo cual el Directorio Empresarial quedó en 1,342 empresas (MiPymes).

La muestra de la base de datos está constituida por 400 MiPymes del estado de Aguascalientes. La técnica de recolección de la información que se aplicó fue una encuesta personal autoadministrada y dirigida a los gerentes de las empresas. En la muestra utilizada no se incluyeron aquellas empresas que tenían menos de cinco trabajadores. 
El trabajo de campo se llevó a cabo durante los meses de septiembre a diciembre de 2008. Para el diseño general de la muestra se consideraron los principios del muestreo estratificado en poblaciones finitas, segmentando en función del tamaño de la empresa y su actividad empresarial. El error muestral obtenido fue $\pm 4.1 \%$ con un grado de fiabilidad de $95 \%$.

\subsection{Medición de las variables}

\section{Cultura empresarial}

Se ha considerado oportuno tomar como base para este trabajo el modelo desarrollado por Cameron y Quinn (1999) para "diagnosticar y cambiar la cultura organizacional". Estos autores se basan en el "Competing Values Framework” propuesto por Quinn (1988), para crear lo que denominan "Instrumento para la valoración de la cultura organizacional (Organizacional Cultura Assessment Instrument, OCAI)”, el cual distingue cuatro tipos de cultura: clan, adhocracia, mercado y jerarquía.

Estos cuatro tipos de cultura quedan definidas a partir de dos dimensiones. Una de ellas refleja en qué medida la organización tiene una orientación hacia el control, la estabilidad y el orden. Esta dimensión varía desde aquellas organizaciones o unidades mecánicas que enfatizan la estabilidad, lo predecible y el orden, hasta aquellas otras que son orgánicas con altos niveles de flexibilidad, cambio y capacidad de adaptación. La segunda dimensión manifiesta la orientación hacia el interior (integración de sus unidades) o el exterior de la empresa (potenciando la diferenciación y rivalidad).

Cruzando estas dos variables se obtienen los cuatro tipos de cultura anteriores (véase figura 1).

La cultura clan es propia de empresas que buscan el control interno pero con flexibilidad preocupados por sus empleados y mostrando sensibilidad a los clientes.

La cultura de adhocracia se encuentra en aquellas empresas que se centran en aspectos externos, pero buscando un alto grado de flexibilidad e innovación. 
La cultura de mercado aparece en aquellas organizaciones que se centran en el exterior pero necesitan control y estabilidad interna.

La cultura de jerarquía se centra en aspectos internos requiriendo control y estabilidad.

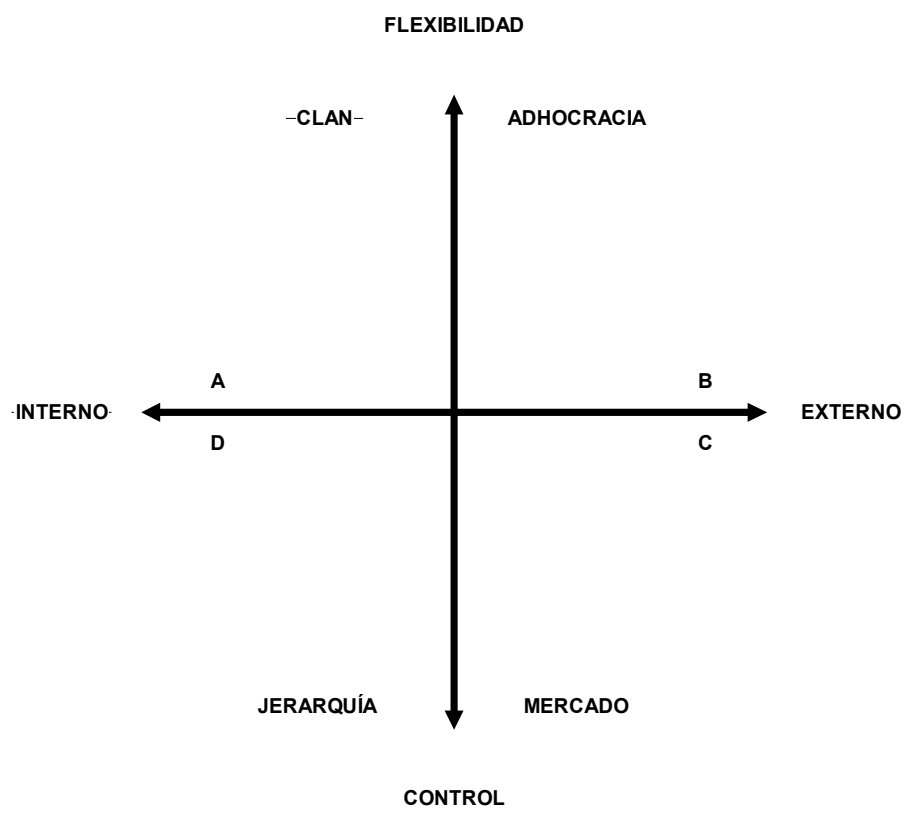

Figura 1

Tipos de cultura según el modelo de Cameron y Quinn (1999)

Cameron y Quinn (1999) defienden que en cualquier organización se encuentran rasgos de los cuatro tipos de cultura, aunque lo frecuente es que haya un predominio de alguna o algunas de ellas.

\section{Rendimiento}

Las variables de rendimiento utilizadas se basan en la clasificación propuesta por Quinn y Rohrbaugh (1983), que establecen un marco para el análisis organizacional y señalan que el criterio de eficiencia organizacional tiene tres ejes o dimensiones. Otros trabajos que han utilizado esta clasificación son: Miron et al. (2004) y Brockman y Morgan (2003). 
La primera relacionada con el enfoque de la organización, desde un punto de vista interno (basado en una visión micro sobre el buen entendimiento y el desarrollo del personal) a uno externo (énfasis a nivel macro del éxito de la empresa).

La segunda, se centra en la estructura organizacional, haciendo hincapié desde la estabilidad hasta la flexibilidad.

Y la tercera dimensión se relaciona con los medios y fines organizacionales. De la combinación de estas tres dimensiones surgen cuatro modelos:

- Modelo de procesos internos: centrado en el control desde el punto de vista interno, dando especial importancia a la comunicación de información, y considerando como fines la estabilidad y el control.

- Modelo de sistema ABIerto: enfocado hacia la flexibilidad desde un punto de vista externo, planteando como principales objetivos el crecimiento, la adquisición de recursos y el apoyo externo.

- Modelo Racional: dirigido hacia el control desde un punto de vista externo, dando especial importancia a los criterios de eficiencia y productividad.

- Modelo de Relaciones humanas: se centra en la flexibilidad desde un punto de vista interno, planteando como principal objetivo el desarrollo de los recursos humanos.

Para valorar los distintos modelos se utilizan 12 ítemes relacionados en el Anexo (tres ítemes por cada modelo) con una escala tipo Likert de 1 a 5 . La variable que representa cada modelo se construye a partir de la media aritmética de los tres ítemes, resultando un rango teórico de 1 a 5 .

Para validar estas medidas se verifica la fiabilidad de las escalas (Alpha de Cronbach), y se comprueba a través de un análisis factorial, que los indicadores anteriores se resumen en un solo factor, capaz de reflejar adecuadamente el rendimiento considerado. Los estadísticos indican la validez de los modelos propuestos. 
Variables de control:

TAMAÑo. Esta variable se midió a través del número medio de empleados del año 2008.

EDAD. Medida a través del número de años transcurridos desde la constitución o inicio de actividad.

\section{ANÁLISIS DE LOS RESULTADOS}

\section{Análisis univariante}

En primer lugar se analizará la tipología existente de cultura empresarial de la muestra de MiPymes del estado de Aguascalientes, con el objeto de conocer la cultura predominante en este contexto. La tabla 1 muestra que la Cultura Clan es la que predomina entre las empresas con 39.15\%, le sigue la Cultura de Mercado con 20.97\%, la Cultura Jerárquica con 20.45\% y, por último, la Adhocrática con sólo 19.70\%.

Si se toma en cuenta el tamaño y la edad de la empresa, la tabla 1 muestra que existen diferencias significativas, respecto a:

- La Cultura Clan predomina más en las pequeñas empresas (45.47\%) que en las Micro (36.06\%) y en las Medianas (35.41\%).

- La Cultura Adhocrática predomina más en las Microempresas (21,12\%), que en las Medianas (18.80\%) y en las Pequeñas (17.53\%).

- La Cultura Jerárquica tiene un mayor grado de predominio entre las empresas Medianas (24.26\%), que en las Micro (20.93\%) y en las Pequeñas (18.55\%).

- La Cultura de Mercado está más posicionada entre las Medianas empresas (21.72\%), que en las Micro (20.05\%) y en las Pequeñas (18.92\%).

- Finalmente, la Cultura Adhocrática tiene una mayor presencia en las empresas más jóvenes (21.26\%) frente a las maduras (18.47\%). 
TABLA 1. Cultura Empresarial (\%)

\begin{tabular}{|l|c|l|l|l|l|l|}
\hline & Microempresas & Pequeñas & Medianas & $\begin{array}{l}\text { Jóvenes } \\
(10 \text { años })\end{array}$ & $\begin{array}{c}\text { Maduras } \\
(>10 \\
\text { años })\end{array}$ & Total \\
\hline - Cultura Clan & $36,06^{* * *}$ & $45,47^{* * *}$ & $35,41^{* * *}$ & 37,48 & 40,46 & 39,15 \\
\hline - Cultura Ad-Hoc & $21,12^{* * *}$ & $17,53^{* * *}$ & $18,80^{* * *}$ & $21,26^{* * *}$ & $18,47^{* * *}$ & 19,70 \\
\hline $\begin{array}{l}\text { - Cultura } \\
\text { Jerárquica }\end{array}$ & $20,93^{* *}$ & $18,55^{* *}$ & $24,26^{* *}$ & 20,51 & 20,40 & 20,45 \\
\hline $\begin{array}{l}\text { - Cultura de } \\
\text { Mercado }\end{array}$ & $20,05^{*}$ & $18,92^{*}$ & $21,72^{*}$ & 21,01 & 20,94 & 20,97 \\
\hline
\end{tabular}

Diferencias estadísticamente significativas: $\left({ }^{*}\right): \mathrm{p} \square 0.1 ;\left(^{* *}\right)$ : p<0.05; $\left(^{* * *}\right): \mathrm{p} \square 0.01$

\section{Análisis multivariante y contrastación de la hipótesis de investigación}

Para verificar el efecto de la cultura empresarial sobre el rendimiento de las MiPymes, hipótesis de investigación planteada, se utilizó una regresión lineal por MCO a través del siguiente modelo:

$$
\text { Rendimiento }_{\mathrm{i}}=\mathrm{b}_{\mathrm{o}}+\mathrm{b}_{1} \cdot \text { Cultura }_{\mathrm{i}}+\mathrm{b}_{2} \text { Tamaño }+\mathrm{b}_{3} \text { Edad }+\varepsilon_{\mathrm{i}}
$$

Donde, Rendimiento ${ }_{\mathrm{i}}$ corresponde a los modelos de procesos internos, sistema abierto, racional y recursos humanos, y rendimiento global. Cultura ${ }_{\mathrm{i}}$ identifica los cuatro tipo de cultura considerada (Clan, Adhocrática, Jerárquica y Mercado). Tamaño, número medio de empleados y Edad, años de existencia de la empresa.

Se estimó un modelo para cada tipo de cultura en relación con las medidas de rendimiento utilizadas (ver tabla 2). 
TABLA 2. Cultura y rendimiento

Número de observaciones: 400

\begin{tabular}{|c|c|c|c|c|c|}
\hline & \multicolumn{4}{|c|}{ Modelos de Rendimiento } & \multirow[b]{2}{*}{$\begin{array}{l}\text { Rendimiento } \\
\text { global }\end{array}$} \\
\hline & $\begin{array}{l}\text { Procesos } \\
\text { internos }\end{array}$ & $\begin{array}{l}\text { Sistema } \\
\text { abierto }\end{array}$ & Racional & $\begin{array}{l}\text { Relaciones } \\
\text { humanas }\end{array}$ & \\
\hline $\begin{array}{l}\text { Cultura } \\
\text { Clan } \\
\text { Tamaño } \\
\text { Edad }\end{array}$ & $\begin{array}{l}\mathbf{0 . 1 3 4 * * *} \\
(2.729) \\
0.190 * * * \\
(3.795) \\
-0.080 \\
(-1.603)\end{array}$ & $\begin{array}{l}\mathbf{0 . 1 0 4} * * \\
(2.122) \\
0.194 * * * \\
(3.872) \\
-0.102 * * \\
(-2.045)\end{array}$ & $\begin{array}{l}\mathbf{0 . 1 8 4} * * * \\
(3.889) \\
0.285 * * * \\
(5.909) \\
-0.163 * * * \\
(-3.385)\end{array}$ & $\begin{array}{l}\mathbf{0 . 1 6 7 * * *} \\
(3.433) \\
0.209 * * * \\
(4.217) \\
-0.046 \\
(-0.926)\end{array}$ & $\begin{array}{l}\mathbf{0 . 1 7 8} * * * \\
(3.699) \\
0.264 * * * \\
(5.404) \\
-0.119 * * \\
(-2.453)\end{array}$ \\
\hline $\begin{array}{l}\text { VIF más alto } \\
\mathrm{F} \\
\mathrm{R}^{2} \text { ajustado }\end{array}$ & $\begin{array}{l}1.041 \\
7.072 * * * \\
0.044\end{array}$ & $\begin{array}{l}1.041 \\
6.733 * * * \\
0.41\end{array}$ & $\begin{array}{l}1.041 \\
17.144 * * * \\
0.110\end{array}$ & $\begin{array}{l}1.041 \\
9.159^{* * *} \\
0.058\end{array}$ & $\begin{array}{l}1.041 \\
14.090 * * * \\
0.090\end{array}$ \\
\hline $\begin{array}{l}\text { Cultura } \\
\text { Adhocrática } \\
\text { Tamaño } \\
\text { Edad }\end{array}$ & $\begin{array}{l}-0.041 \\
(-0.820) \\
0.104 * * \\
(2.028) \\
-0.076 \\
(-1.478)\end{array}$ & $\begin{array}{l}-0.042 \\
(-0.832) \\
0.082 \\
(1.606) \\
-0.092 * \\
(-1.783)\end{array}$ & $\begin{array}{l}\mathbf{- 0 . 0 8 3 *} \\
(-1.683) \\
0.175^{* * *} \\
(3.473) \\
-0.163 \\
(-3.225)\end{array}$ & $\begin{array}{l}\mathbf{- 0 . 0 9 9 * *} \\
(-1.973) \\
0.104 * * \\
(2.035) \\
-0.046 \\
(-0.888)\end{array}$ & $\begin{array}{l}-0.081 \\
(-1.615) \\
0.141 * * * \\
(2.764) \\
-0.116 * * \\
(-2-266)\end{array}$ \\
\hline $\begin{array}{l}\text { VIF más alto } \\
\mathrm{F} \\
\mathrm{R}^{2} \text { ajustado }\end{array}$ & $\begin{array}{l}1.068 \\
1.936 \\
0.007\end{array}$ & $\begin{array}{l}1.068 \\
1.741 \\
0.006\end{array}$ & $\begin{array}{l}1.068 \\
6.879 * * * \\
0.042\end{array}$ & $\begin{array}{l}1.068 \\
2.782^{* *} \\
0.013\end{array}$ & $\begin{array}{l}1.068 \\
4.267 * * * \\
0.24\end{array}$ \\
\hline $\begin{array}{l}\text { Cultura } \\
\text { Mercado } \\
\text { Tamaño } \\
\text { Edad }\end{array}$ & $\begin{array}{l}-0.039 \\
(-0.785) \\
0.109 * * \\
(2.117) \\
-0.070 \\
(-1.373)\end{array}$ & $\begin{array}{l}0.010 \\
(0.190) \\
0.083 \\
(1.610) \\
-0.088^{*} \\
(-1.707)\end{array}$ & $\begin{array}{l}0.019 \\
(0.379) \\
0.176^{* * *} \\
(3.472) \\
-0.155^{* * *} \\
(-3.061)\end{array}$ & $\begin{array}{l}-0.041 \\
(-0.817) \\
0.111^{* *} \\
(2.156) \\
-0.033 \\
(-0.651)\end{array}$ & $\begin{array}{l}-0.014 \\
(-0.286) \\
0.145^{* * *} \\
(2.823) \\
-0.107 * * \\
(-2.089)\end{array}$ \\
\hline $\begin{array}{l}\text { VIF más alto } \\
\mathrm{F} \\
\mathrm{R}^{2} \text { ajustado }\end{array}$ & $\begin{array}{l}1.063 \\
1.917 \\
0.007\end{array}$ & $\begin{array}{l}1.063 \\
1.520 \\
0.004\end{array}$ & $\begin{array}{l}1.063 \\
5.943 * * * \\
0.036\end{array}$ & $\begin{array}{l}1.063 \\
1.696 \\
0.005\end{array}$ & $\begin{array}{l}1.063 \\
3.403 * * \\
0.018\end{array}$ \\
\hline $\begin{array}{l}\text { Cultura } \\
\text { Jerárquica } \\
\text { Tamaño } \\
\text { Edad }\end{array}$ & $\begin{array}{l}\mathbf{- 0 . 1 6 5} * * * \\
(-3.337) \\
0.100 * * \\
(1.974) \\
-0.060 \\
(-1.176) \\
\end{array}$ & $\begin{array}{l}\mathbf{- 0 . 1 5 5 * * *} \\
(-3.141) \\
0.079 \\
(1.549) \\
-0.076 \\
(-1.498) \\
\end{array}$ & $\begin{array}{l}\mathbf{- 0 . 2 6 0} * * * \\
(-5.477) \\
0.169 * * * \\
(3.468) \\
-0.135 * * * \\
(-2.771) \\
\end{array}$ & $\begin{array}{l}\mathbf{- 0 . 1 7 8 * * *} \\
(-3.614) \\
0.101 * * \\
(2.006) \\
-0.022 \\
(-0.431) \\
\end{array}$ & $\begin{array}{l}\mathbf{- 0 . 2 2 9} * * * \\
(-4.715) \\
0.136 * * * \\
(2.733) \\
-0.090 * \\
(-1.816) \\
\end{array}$ \\
\hline $\begin{array}{l}\text { VIF más alto } \\
\mathrm{F} \\
\mathrm{R}^{2} \text { ajustado }\end{array}$ & $\begin{array}{l}1.061 \\
5.469 * * * \\
0.033\end{array}$ & $\begin{array}{l}1.061 \\
4.834 * * * \\
0.028\end{array}$ & $\begin{array}{l}1.061 \\
16.337 * * * \\
0.103\end{array}$ & $\begin{array}{l}1.061 \\
5.872 * * * \\
0.035\end{array}$ & $\begin{array}{l}1.061 \\
10.977 * * * \\
0.070\end{array}$ \\
\hline
\end{tabular}

Debajo de cada coeficiente estandarizado, entre paréntesis, valor del estadístico t-student

* $\mathrm{p} \leq 0.1 ; * * \quad \mathrm{p} \leq 0.05 ; * * * \quad \mathrm{p} \leq 0.01$ 
Se observó en todos los modelos que las variables independientes tienen un factor de la inflación de la varianza (VIF) cercano a 1, por lo que se descartó la presencia de multicolinealidad.

Los resultados muestran que el tipo de cultura implantado en el seno de las Pyme influyen en su rendimiento, por lo que se confirma la hipótesis de trabajo planteada.

En relación con la medida de rendimiento global utilizada, los resultados indican de forma muy significativa $(\mathrm{p}<0.01)$, por una parte, que una mayor presencia de cultura clan en la Pyme ejerce una influencia positiva en su rendimiento (coeficiente estandarizado $=0.178$ ), y por otra, que una mayor presencia de cultura jerárquica ejerce una influencia negativa sobre su rendimiento (coeficiente estandarizado $=-0.229$ ). No se obtuvo, sin embargo, evidencia en relación a la cultura adhocrática y de mercado.

Si se analiza con más detalle las diferentes medidas de rendimiento según el modelo de Quinn y Rohrbaugh (1983), se puede apreciar que:

La cultura clan ejerce una influencia muy significativa en todos los modelos, especialmente en el modelo racional (coeficiente estandarizado = o.184), al igual que sucede con la cultura jerárquica con signo negativo, pero con impacto mayor.

En efecto, la cultura jerárquica principalmente ejerce una influencia negativa sobre el modelo de rendimiento racional (coeficiente estandarizado $=-0.260$ ). Recordemos que el modelo racional hace énfasis en la eficiencia y productividad de la empresa.

Adicionalmente, y respecto a la cultura adhocrática, encontramos un resultado contrario a lo esperado. La cultura adhocrática implica una mayor orientación hacia la innovación y sería esperar una relación positiva. Sin embargo, en el modelo racional $(\mathrm{p}<0.1)$ y en el modelo de relaciones humanas $(\mathrm{p}<0.05)$ los resultados muestran un signo negativo. 


\section{CONCLUSIONES}

La cultura empresarial constituye un tópico relevante en la investigación de la empresa. Sin embargo, el estudio de la cultura particularizada en las empresas de reducida dimensión ha recibido relativamente poca atención por parte de los investigadores. Al objeto de contribuir con nueva evidencia empírica, este trabajo analiza la relación entre cultura empresarial y el rendimiento, utilizando una muestra de 400 MiPymes del estado de Aguascalientes (México).

Para medir la cultura empresarial hemos seguido la metodología propuesta por Cameron y Quinn (1999), que distinguen cuatro tipos de cultura: Mercado, Jerárquica, Clan y Adhocrática, y para medir el rendimiento se ha utilizado la metodología propuesta por Quinn y Rohrbaugh (1983) que identifican cuatro modelos de rendimiento: procesos internos, sistema abierto, racional y relaciones humanas.

Los resultados obtenidos vienen a señalar que la cultura empresarial ejerce una importante influencia en el rendimiento de la MiPyme en el contexto de México, confirmando la hipótesis planteada. Por una parte, la evidencia empírica señala una relación positiva entre la cultura clan y el rendimiento. La cultura clan conlleva actitudes orientadas hacia el control interno pero con flexibilidad, preocupados por sus empleados y mostrando sensibilidad a los clientes, y donde el éxito se basa en el desarrollo humano, el trabajo en equipo, el compromiso e interés por los trabajadores. La empresa es como una gran familia. Por otra parte, y con signo contrario, los resultados muestran que la cultura jerárquica ejerce una influencia negativa en la MiPyme.

La cultura jerárquica se centra en aspectos internos requiriendo control y estabilidad. La empresa está muy jerarquizada, formalizada y estructurada. Para cualquier actividad existen normas y procedimientos. El éxito se basa en la eficiencia. Asimismo, se encontró que en las MiPyme analizadas existe una baja orientación hacia la cultura adhocrática, más relacionada con una actitud dinámica y emprendedora, asumiendo riesgos hacia la innovación, basando su éxito en desarrollar productos únicos 
y novedosos. La innovación permite a las empresas lograr ventajas competitivas sostenibles en el tiempo (Vermeulen, 2004) y representa un elemento determinante del crecimiento económico (Cheng y Tao, 1999).

Además, surge un signo negativo respecto de la cultura adhocrática en los modelos de rendimiento racional y de relaciones humanas y contrarios a lo esperado. La explicación de este comportamiento puede obedecer a que en el corto plazo la adopción de una cultura orientada hacia la innovación pueda provocar una menor rentabilidad o margen y generar tensiones entre sus recursos humanos si éstos no cuentan con la cualificación necesaria para llevarlas a cabo. No obstante, estos comentarios precisan de un mayor desarrollo para su contrastación empírica, lo que abre una sugerente extensión de la investigación dirigida a conocer las causas de este signo negativo.

Este trabajo contribuye con nueva evidencia empírica al cuerpo de la literatura sobre Teoría de los Recursos y Capacidades, mostrando la necesidad que tiene la MiPyme de establecer una cultura empresarial que las posicione hacia un correcto equilibrio de su crecimiento y rentabilidad. Adicionalmente, no se puede olvidar que si motivamos a las MiPyme para que desarrollen un mayor grado de innovación para fortalecer su competitividad, debemos asegurarnos cuáles son los rendimientos esperados frente a las empresas que tienen una actitud más conservadora o jerárquica.

Las implicaciones de este trabajo, por tanto, son relevantes. Los resultados pueden ser útiles a los propietarios y directivos de las empresas. La cultura empresarial es un factor clave que puede ayudar a las empresas a mejorar su rendimiento, por lo que reorientar su actitud cultural puede llegar a convertirse en una ventaja competitiva. El reto en México recae en consolidar una cultura empresarial realizando por una lado, los cambios necesarios, y por otro lado, que se superen y queden en el pasado los patrones de conducta tradicionales, donde los procesos vayan en dirección al desarrollo de las empresas, creando ventajas competitivas y nuevas oportunidades y prestando atención a los valores, costumbres y al entorno específico de cada empresa. 
También se espera que los resultados puedan ser útiles para los organismos públicos a la hora de diseñar sus estrategias para fortalecer el tejido empresarial y mejorar la supervivencia de las empresas.

\section{BIBLIOGRAFÍA}

Brockman, B. y R.M. Morgan (2003) "The role of existing knowledge in new product innovativeness and performance", Decision Science, spring, Vol. 34 (2), pp. 385-420.

Burt, S. y L. Sparks (1994) "Structural changes in grocery retailing in Great Britain: a discount re-orientation", The International Review of Retail, Distribution and Consumer Research, núm. 3, vol. 1, pp. 195-217.

Cameron, K. S. y R.E. Quinn (1999) Diagnosing and changing organizational culture. Base on the competing values framework, AddisonWesley. Reading.

Cheng, L. K. y Z. Tao (1999) "The impact of public policies on innovation and imitation: the role of R\&D technology in growth models", International Economic Review, Vol. 40, $\mathrm{n}^{0}$ 1, pp. 187-207.

De Long, D., y L. Fahey (2000) "Diagnosing cultural barriers to knowledge management", Academy of Management Executive, núm. 14, vol. 4, pp. 113-127.

Deal, T.E. y A.A. Kennedy (1982) Corporate cultures, Reading, MA: Addison-Wesley.

Denison, D.R. y A.K. Mishra (1995) "Toward a theory of organizational culture and effectiveness", Organization Science, núm. 6, vol.2, pp. 204-223.

Denison, D.R., y G.M. Spreitzer (1991) "Organizational culture and organizational development: A competing values approach", Research in Organizational Change and Development, núm. 5, pp. 1-21.

García Pérez de Lema, D. (2005) Estrategia e innovación de la Pyme industrial en España, Asociación Española de Contabilidad y Administración de Empresas, Madrid. 
García Pérez de Lema, D. y M.C. Martínez Serna (2009) Innovación y cultura empresarial de las Mipyme (Micro, Pequeña y Mediana Empresa) estado de Aguascalientes, Universidad Autónoma de Aguascalientes y Universidad Politécnica de Cartagena, México: Aguascalientes.

Gordon, G. G. y N. Di Tomaso (1992) "Predicting corporate performance from organizational culture", Journal of Management Studies; núm. 29, pp. 783-799.

Jeuchter, W.M., C. Fisher, y R.J. Alford (1998) "Five conditions for high performance culture", Training and Development, núm. 52, vol. 5, pp. 63-67.

Kotter, J.P. y J.L. Heskett (1992) Corporate Culture and Performance, The Free Press, New York NY.

Leal, A. (1991) Conocer la cultura de las organizaciones: una base para la estrategia y el cambio, Actualidad Editorial, Madrid.

Leal, A. (1991) "Cultura organizativa y orientación al mercado: un análisis multisectorial en pyme”, XIV Congreso Acede. Murcia.

Maani, K.E., M.S. Puga Putterill, y D.G. Sluti (1994) "Empirical analysis of Quality Improvement in Manufacturing. Asia Pacific”, Journal of Quality Management, núm. 3, vol. 1, pp. 5-23.

March, J. G. (1991) "Exploration and exploitation in organizational learning”, Annual Review of Sociology; núm. 14, pp. 319-340.

Miron, E.; M. Erez, y E. Naveh (2004) "Do personal characteristics and cultural values that promote innovation, quality, and efficiency compete or complement each other?", Journal of Organizational Behavior, Vol. 25 Issue 2, pp. 175-200.

Petty, M.M., N.A. Beadles II, C.M. Lowery (1995) "Relationship between Organizational Culture and Organizational Performance“, Psychological Reports, núm. 76, vol. 2, pp. 483-492.

Quinn, R.E., y J. Rohrbaugh (1983) "A spatial model of effectiveness criteria: towards a competing values approach to organizational analysis", Management Science, núm. 29, vol. 3, pp. 363-377.

Rosenthal, J., y M. Masarech (2003) "High-performance cultures: how values can drive business results", Journal of Organizational Excellence, núm. 22 vol. 2, pp. 3-18. 
Schneider, B. (1990) Organizational Climate and Culture, Jossey-Basss, San Francisco, CA.

Siehl, C., y J. Martin (1998) Measuring Organizational Culture: Mixing Qualitative and Quantitative Methods, in Jones, M.O, et al. (Eds), Inside Organizations: Understanding the Human Dimension, Sage Publications, Newbury Park, CA, pp. 79-103.

Sonrensen, J. (2002) "The strength of corporate culture and the reliability of firm performance”, Administrative Science Quarterly, March 47(1), pp. 70-91.

Tushman, M. L., y Ch.A. O'Reilly III (2002) Winning through innovation. A practical guide to leading organizational change and renewal, Harvard Business School Press. Boston.

Tushman, M.L., W. Smith, R. Wood, G. Westerman, y Ch. O’Reilly (2002) "Innovation Streams and Ambidextrous Organizational Designs: On Building Dynamic Capabilities", December 2, Disponible en: http://web.mit.edu/sloan/osg-seminar/fo2_docs/ TushmanEtAl_2002.pdf.

Vermeulen, P. (2004) "Managing Product Innovation in Financial Service Firms”, European Management Journal 22(1), pp. 43-50. 


\section{ANEXO CUESTIONARIO}

1. Para cada una de las cuatro cuestiones siguientes, reparta 100 puntos entre las 4 respuestas $(a, b, c, d)$ que reflejan lo más apegado a su realidad en los distintos aspectos de la cultura actual de su empresa:

\section{Defina SU EMPRESA:}

a) Es como una gran familia. La gente comparte mucho con los demás

b) Es una entidad muy dinámica y emprendedora. La gente está dispuesta a apostar por sus ideas y asumir riesgos

c) Es una entidad orientada a resultados. La gente es muy competitiva y enfocada fundamentalmente a conseguir los objetivos

d) Es una entidad muy jerarquizada, formalizada y estructurada. Para cualquier actividad existen procedimientos y normas previamente definidos

TOTAL

Actual

Defina el ESTILO DE DIRECCIÓN EN SU EMPRESA:

a) Se caracteriza por promover el trabajo en equipo, el consenso y la participación

b) Se caracteriza por promover la iniciativa del individuo, la asunción de riesgos, la innovación y la singularidad

c) Se caracteriza por promover la competitividad agresiva y la consecución de objetivos ambiciosos

d) Se caracteriza por promover la seguridad en el empleo, la permanencia en el puesto y la existencia de poca incertidumbre

TOTAL

Defina los VALORES COMPARTIDOS POR EL PERSONAL DE SU EMPRESA:

Actual

a) Los valores compartidos son la lealtad, el compromiso con la empresa, la confianza mutual y el trabajo en grupo

b) Los valores compartidos son el compromiso con la innovación y el cambio continuo

c) Los valores compartidos son la agresividad, el espíritu ganador y la consecución de los objetivos previstos

d) Los valores compartidos con la empresa son el respeto por las normas y políticas formales y el cumplimiento con la jerarquía

TOTAL

100

Defina en qué basa EL ÉXITO DE SU EMPRESA:

Actual

a) El éxito se basa en el trabajo en equipo, el compromiso e interés por los trabajadores

b) El éxito se basa en el desarrollo de productos únicos y novedosos. Ser líder en productos e innovación 
c) El éxito se basa en la penetración en el mercado y la cuota de mercado. Ser el número uno respecto a los competidores

d) El éxito se basa en la eficiencia. La adecuada programación de la producción y los bajos costes son aspectos críticos

\begin{tabular}{|c|c|c|c|c|}
\hline TOTAL & & & & \\
\hline $\begin{array}{l}\text { 2. Indique cuál ha sido la evolución de los siguientes } \\
\text { aspectos en su empresa en los dos últimos años }\end{array}$ & $\begin{array}{l}\text { Muy } \\
\text { desfavo }\end{array}$ & & favo & Muy \\
\hline -Calidad del producto / servicio & 1 & 3 & 4 & 5 \\
\hline -Eficiencia de los procesos operativos internos & 2 & 3 & 4 & 5 \\
\hline -Organización de las tareas del personal & 2 & 3 & 4 & 5 \\
\hline -Satisfacción de los clientes & 1 & 3 & 4 & 5 \\
\hline $\begin{array}{l}\text {-Rapidez de adaptación a las necesidades de los } \\
\text { mercados }\end{array}$ & 1 & 3 & 4 & 5 \\
\hline -Imagen de la empresa y de sus productos / servicios & 1 & 3 & 4 & 5 \\
\hline -Incremento de la cuota de mercado & 1 & 3 & 4 & 5 \\
\hline -Incremento de la rentabilidad & 1 & 3 & 4 & 5 \\
\hline -Incremento de la productividad & 1 & 3 & 4 & 5 \\
\hline -Motivación / satisfacción de los trabajadores & 1 & 3 & 4 & 5 \\
\hline $\begin{array}{l}\text { - Reducción de la rotación de personal (abandono } \\
\text { voluntario trabajadores) }\end{array}$ & 1 & 3 & 4 & 5 \\
\hline -Reducción del ausentismo laboral & 1 & 3 & 4 & 5 \\
\hline
\end{tabular}

